Knowledge Warehouse: An Architectural Integration of Knowledge Management, Decision Support, Artificial Intelligence and Data Warehousing

By: Hamid R. Nemati, David M. Steiger, Lakshmi S. Iyer, and Richard T. Herschel.

Nemati, H.R., D. Steiger, L.S. Iyer, and R.T. Herschel. (2002). "Knowledge Warehouse: An Architectural Integration of Knowledge Management, Decision Support, Artificial Intelligence and Data Warehousing," Decision Support Systems, 33 (2), 143-161.

Made available courtesy of Elsevier: http://dx.doi.org/10.1016/S0167-9236(01)00141-5

***;( Elsevier. Reprinted with permission. No further reproduction is authorized without written permission from Elsevier. This version of the document is not the version of record. Figures and/or pictures may be missing from this format of the document. ***

c) (i) (9)

EY NC NO This work is licensed under a Creative Commons AttributionNonCommercial-NoDerivatives 4.0 International License.

\begin{abstract}
:
Decision support systems (DSS) are becoming increasingly more critical to the daily operation of organizations. Data warehousing, an integral part of this, provides an infrastructure that enables businesses to extract, cleanse, and store vast amounts of data. The basic purpose of a data warehouse is to empower the knowledge workers with information that allows them to make decisions based on a solid foundation of fact. However, only a fraction of the needed information exists on computers; the vast majority of a firm's intellectual assets exist as knowledge in the minds of its employees. What is needed is a new generation of knowledge-enabled systems that provides the infrastructure needed to capture, cleanse, store, organize, leverage, and disseminate not only data and information but also the knowledge of the firm. The purpose of this paper is to propose, as an extension to the data warehouse model, a knowledge warehouse (KW) architecture that will not only facilitate the capturing and coding of knowledge but also enhance the retrieval and sharing of knowledge across the organization. The knowledge warehouse proposed here suggests a different direction for DSS in the next decade. This new direction is based on an expanded purpose of DSS. That is, the purpose of DSS in knowledge improvement. This expanded purpose of DSS also suggests that the effectiveness of a DS will, in the future, be measured based on how well it promotes and enhances knowledge, how well it improves the mental model(s) and understanding of the decision maker(s) and thereby how well it improves his/her decision making.
\end{abstract}

Keywords: Knowledge warehouse | Data warehouse | Knowledge management | Decision support systems | Data mining | Intelligent analysis | Model analysis

Article:

1. Introduction 
The complexities of decisions in the information age compel every manager to utilize information analysis tools for supporting business decisions. Over the last three decades, the organizational role of information technology has evolved from efficiently processing large amounts of batch transactions to providing information in support of decision-making activities. This paradigm shift is reflected in the fact that in the 1970s most IS organizations changed their name from "data processing" to "management information systems" [7]. In addition, the variability, interdependency and uncertainty of factors affecting decision-making process are complex. Decision support systems (DSS) are interactive, computer-based systems intended to provide support to the decision makers engaged in solving various semi- to ill-structured problems involving multiple attributes, objectives and goals. Decision support systems are becoming increasingly more critical in the daily operation of organizations. With the evolution of enterprise network computing, client/ server architecture, and a set of significant new information processing concepts, it is now possible for organizations to provide the key people in the firm with access to needed information and the means to utilize that information in a decision support context.

Since the mid-1980s data warehouses have been developed and deployed as an integral part of a modern decision support environment. A data warehouse provides an infrastructure that enables businesses to extract, cleanse, and store vast amounts of corporate data from operational systems for efficient and accurate responses to user queries [26]. A data warehouse empowers knowledge workers with information that allows them to make decisions based on a solid foundation of fact [12]. However, only a fraction of the required knowledge exists on computers; the vast majority of a firm's intellectual assets exist as knowledge in the minds of its employees [48]. Hence, a data warehouse does not necessarily provide adequate support for knowledge intensive queries in an organization. What is needed is a new generation of knowledge enabled systems that provides the infrastructure required to capture, enhance, store, organize, leverage, analyze, and disseminate not only data and information but also knowledge. The existing enterprise-wide data warehouses can be extended to create a knowledge warehouse $(\mathrm{KW})$. This warehouse can be used as a clearinghouse of knowledge to be used throughout the organization by the employees to support their knowledge intensive decision-making activities. The KW can also evolve over time by enhancing the knowledge it contains.

Just as in a data warehouse environment where data mining techniques can be used to discover untapped patterns of data that enable the creation of new information, by extension then, use of technologies such as data warehousing, data mining and other artificial intelligence (AI) technologies can enhance the knowledge creation, storage, dissemination and management processes [2]. However, for an effective knowledge warehouse to become a reality, different types of knowledge (i.e., both tacit and explicit knowledge) and different forms of knowledge (e.g., text streams, binary large objects, production rules, mathematical models, and what-if cases) need to be captured, codified, and cataloged. In addition, this codified knowledge must contain knowledge about itself (meta-knowledge) and must be analyzed to create new knowledge.

The purpose of this paper is to describe the processes required for developing a knowledge warehouse and to propose, as an extension to the data warehouse model, a knowledge warehouse architecture that can facilitate the capturing, coding, retrieval and sharing of knowledge. The KW 
is used to enhance the generation of new knowledge throughout the organization. The primary goal of a $\mathrm{KW}$ is to provide the knowledge worker with an intelligent analysis platform that enhances all phases of the knowledge management process. Just as the emergence of data warehouses a decade ago signaled a new direction for the DSS, we argue that the knowledge warehouse proposed here suggests a new and evolving direction for DSS in the next decade. This new direction is based on an expanded purpose of DSS.

That is, the purpose of DSS in knowledge improvement; i.e., enhanced learning. This expanded purpose of DSS also suggests that the effectiveness of each DSS will, in the future, be measured based on how well it promotes and enhances knowledge, how well it improves the mental model(s) and understanding of the decision maker(s) and thereby how well it improves his/her decision making.

The remainder of this paper is organized into the following sections. In Section 2, we provide some knowledge management background. In Section 3, we discuss how DSS, artificial intelligence (AI) and information technology (IT) can enhance knowledge management. We then present the foundations for the goals and requirements for a $\mathrm{KW}$ in Section 4. In Section 5, we present the proposed KW architecture. In Section 6, we discuss guidelines as how such a warehouse could be implemented. In Section 7, we provide a roadmap for future DSS research based on our proposed architecture. Finally, in Section 8, we provide our summary and conclusions.

\section{Knowledge management}

Knowledge management is the practice of adding actionable value to information by capturing tacit knowledge and converting it to explicit knowledge; by filtering, storing, retrieving and disseminating explicit knowledge; and by creating and testing new knowledge. In this context, tacit knowledge includes the beliefs, perspectives, and mental models so ingrained in an person's mind that they are taken for granted [48]; it consists of subjective expertise, insights and intuitions that a person develops from having been immersed in an activity or profession for an extended period of time. On the other hand, explicit knowledge is knowledge that can be expressed formally using a system of language, symbols, rules, objects, or equations, and can thus be communicated to others; it consists of quantifiable data, written procedures, universal principles, mathematical models, etc. $[10,48,72]$.

New knowledge is created through the synergistic relationship and interplay between tacit and explicit knowledge [48], specifically, through a four-step process of socialization, articulation, integration, and understanding/ internalization (Fig. 1). Socialization is the process of sharing with others the experiences, technical skills, mental models, and other forms of tacit knowledge. For example, apprentices learn a craft not through language, but by working with their masters; i.e., observing, imitating and practicing under the master's tutelage. On-the-job-training provides this mode of sharing tacit knowledge in the business world. OJT is complemented with film clips of the expert performing the task, virtual reality representations, and kinematic analysis (from the field of robotics). 


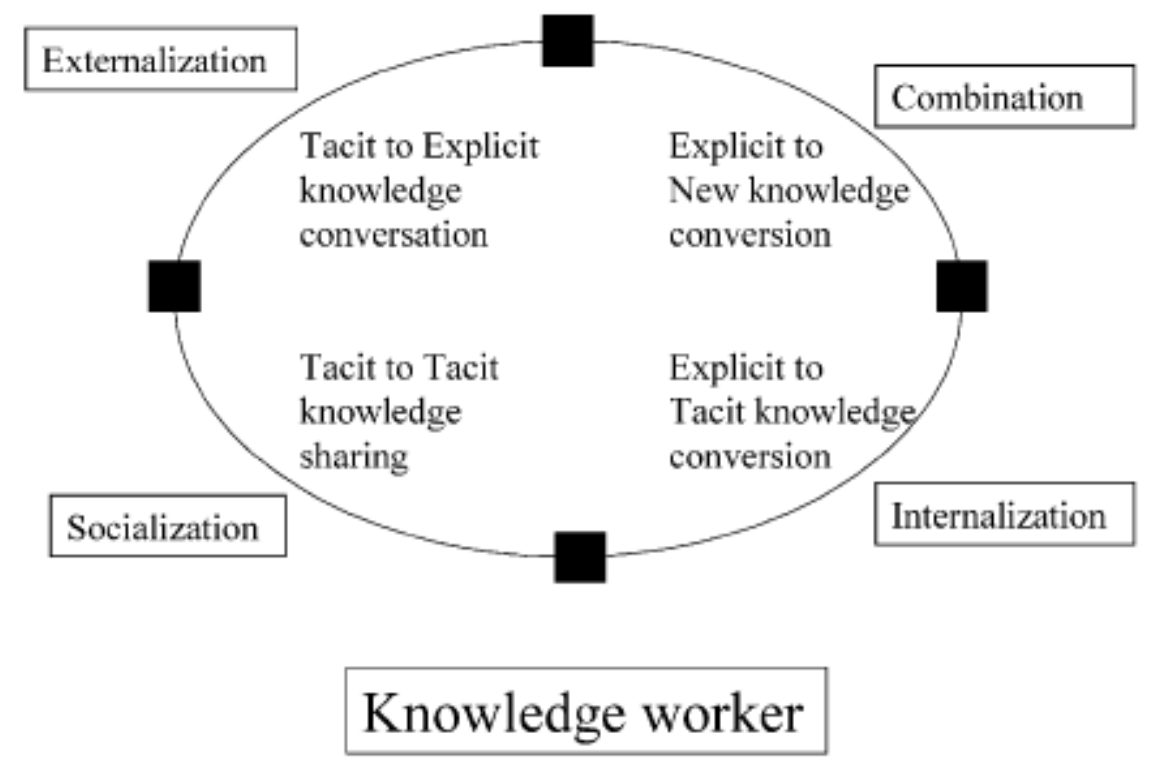

Fig. 1. The knowledge spiral.

Articulation is the process of converting tacit knowledge to explicit knowledge. In the decision making process, articulation may include, but is not limited to, one or more of the following: (1) specifying the purpose of the decision; e.g., to understand how the number and locations of warehouses influence supply costs in a new marketing area, (2) articulating parameters, objective functions, relationships, etc., in a DSS math mathematical model (i.e., building a model), (3) articulating 'what-if' model cases that reflect existing and potential decision making situations, and (4) evaluating the decision alternatives, given the uncertainty in the decision making environment. In other situations (e.g., those requiring the analysis of complicated physical movements), articulation may take the form of kinematic analysis; i.e., attaching sensors to various key appendages and then digitizing and recording the movements of interest. Articulation may also include knowledge extraction in expert systems, determination of causal maps, brainstorming, etc.

Integration is the process of combining several types of explicit knowledge into new patterns and new relations. The Gestalt theory of learning literature states that "all problems with which we may be confronted, and also the solutions of such problems, are matters of relations; not only does our understanding of the problem demand our awareness of certain relations, we cannot solve the problem without discovering certain new relations" [51]. One potentially productive integration of explicit knowledge is the analysis of multiple, related 'what-if' cases of a mathematical model to find new relationships, or meta-models, that determine the key factors of the model and show how these key factors interact to influence the decision [62].

Understanding is the process of testing and validating the new relationships in the proper context, thereby converting them into new tacit knowledge. Perkins's [51] theory of understanding, from the theory of learning literature, suggests that understanding involves the knowledge of three things: the purpose of the analysis (i.e., what the decision maker wants to 
understand), a set of relations or models of the process/system to be understood, and arguments about why the relations/models serve the purpose. Internalization is the process of using the new patterns and relations, together with the arguments of why they fit the purpose, to update and/or extend the decision maker's own tacit knowledge base, thus creating a spiral of learning and knowledge that begins and ends with the individual $[23,48]$.

For a more comprehensive review of tacit to explicit knowledge conversion, please refer to Refs. $[48,11]$.

\section{DSS, IT, and AI support of knowledge management}

DSS, IT, and AI can all be used to enhance knowledge management and its knowledge conversion processes: i.e., tacit to tacit knowledge sharing, tacit to explicit knowledge conversion, explicit knowledge leveraging, and explicit to tacit knowledge conversion. These process enhancements are discussed individually below (Fig. 2).

\subsection{Sharing tacit knowledge}

One of the primary potential applications of information technology to sharing tacit knowledge is the use of digitized filming of the physical demonstration of a process. Once stored, this digitized film clip can be made available on the internet for anytime, anyplace viewing. The film clip can also include slow motion segments of the physical process where applicable, complete with verbal explanations included within the clip to enhance the understanding of the process being demonstrated.

A potential application of artificial intelligence to tacit to tacit knowledge sharing is the use of kinematic analysis of the physical process. Kinematics includes the use of reflective dots and/or sensors attached to the various appendages and joints of the demonstrator to enhance the determination of quick or subtle movements or actions during the demonstrated process; e.g., to detect twisting or turning of the fingers while a master chef kneads bread dough. Once the process is recorded, kinematic analysis software is used to further analyze the relative motion of the appendages and joints; thus, kinematics provides a natural conversion of tacit knowledge to explicit knowledge.

\subsection{Converting tacit knowledge to explicit knowledge}

In tacit to explicit knowledge conversion, the literature of knowledge acquisition in expert systems (ES) provides both guidance and techniques [35]. Knowledge acquisition involves employing various techniques to elicit information (verbal and/or quantitative) from the knowledge worker, interpreting this information (more or less skillfully) in order to infer the underlying knowledge and reasoning processes, and using this interpretation to guide the construction of some model or language that describes (more or less accurately) the knowledge worker's performance [28]. 


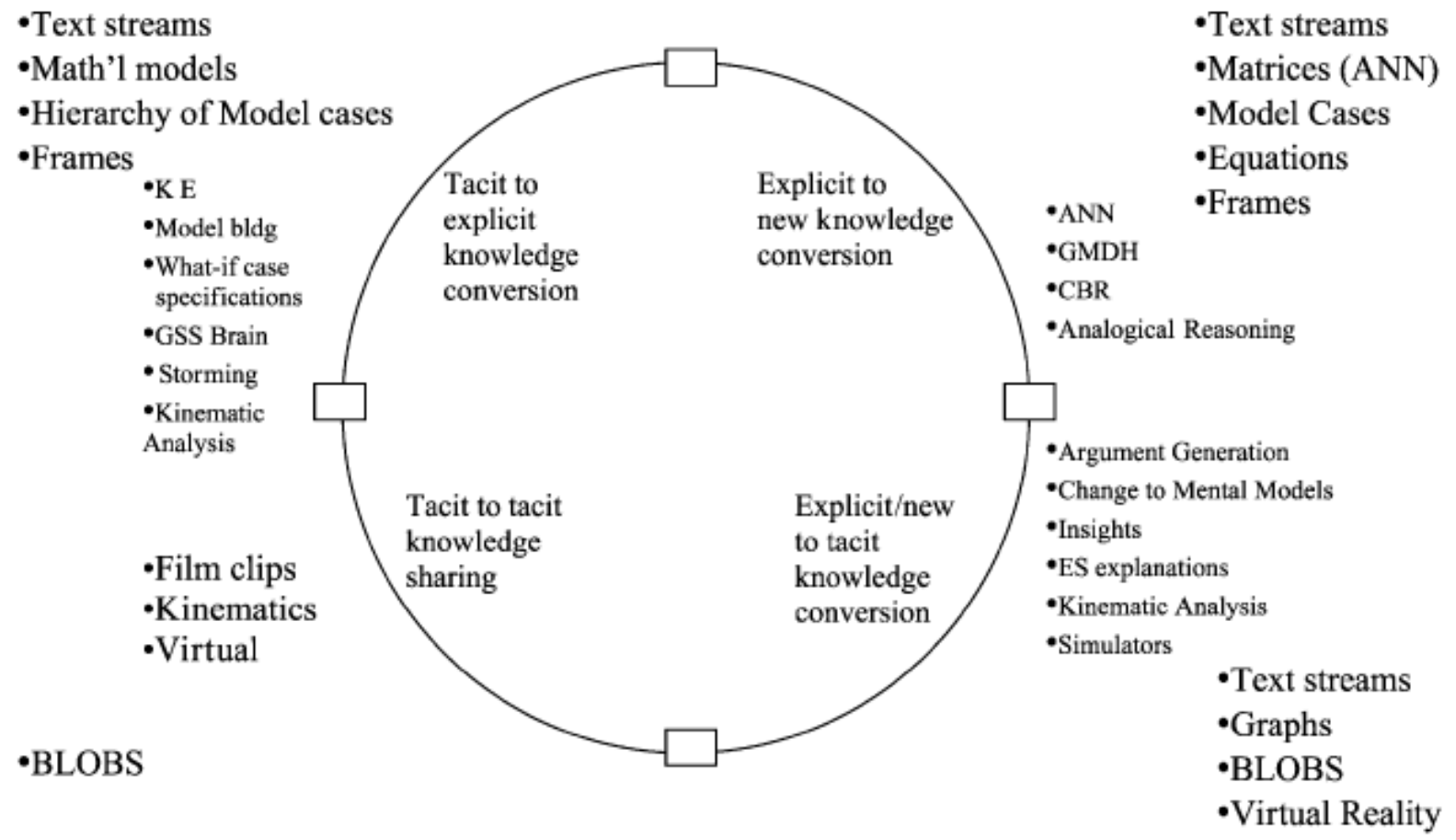

Fig. 2. Technologies and data/knowledge types in knowledge management.

DSS can also enhance the tacit to explicit knowledge conversion through the specification of mathematical models. Specifically, in the model building process (e.g., in linear programming models) the knowledge worker is asked to explicitly specify the goal or objective of the model, the decision variables, and perhaps the relative importance of the decision variables (in the case of a goal programming model). The knowledge worker also explicitly specifies the model constraints in terms of the decision variables, and estimates both the numerical coefficients of the decision variables in each constraint and in the objective function, as well as the right hand side constraint values. The explicit knowledge reflected in these model components (decision variables, coefficients, constraints and objective functions) reflects the tacit knowledge built up over the years of being immersed in the decision making environment. The resulting models may be stored in the form of a set of explicit mathematical inequalities [16], as annotated graphs of arcs and nodes in network flow models [29,64], as a set of arc descriptions [34] or as a condensed canonical model formulation with links to relational tables for instantiation [63].

DSS can also enhance the tacit to explicit knowledge conversion by eliciting one or more what-if cases (i.e., model instances) representing situations that the knowledge worker wants to explore. As the knowledge worker changes one or more model coefficients or right hand side values (e.g., in a linear programming model) to explore its effect on the modeled solution, s/he is estimating ranges of those parameters/values that reflect the actual and/or potential decision making environment represented by the model. That is, the knowledge worker is converting the tacit knowledge of various historical situations and/or decisions into explicit knowledge that can be 
shared with other workers and leveraged to enhance decision making. These multiple, related model instances can be stored, along with their associated solutions, as tuples in a relational database, as objects in an object-oriented database, or as sparse matrices.

Another source of tacit to explicit knowledge conversion occurs in the brainstorming of GSS. GSS brainstorming sessions solicit the participants' ideas and concerns about a stated problem. The ideas are then anonymously relayed (without evaluative comments) to the other participants for their enhancements and modifications, generating a continual stream of related and tangential ideas directed toward solving the stated problem. At some point of time, the session leader directs the participants to stop generating new ideas and start evaluating, again anonymously, a specific idea. The evaluations are given in the form of short lists of things the participant likes about the idea and also short lists of concerns that may hamper implementation. The group then addresses the concerns, evolving toward a valid and implementable solution to the stated problem. The ideas, likes and dislikes of GSS brainstorming sessions are stored as text streams for sharing, processing and future use.

\subsection{Knowledge leveraging: converting explicit knowledge to new knowledge}

Once a knowledge worker's tacit knowledge is converted to explicit knowledge and stored in an appropriate (computer readable) form, it can be leveraged by making it available to others when and where they need it. In addition, analyzing explicit knowledge to produce new knowledge can further leverage it.

For example, explicit knowledge generated from GSS brainstorming sessions and stored as text streams can be analyzed by text mining software, a form of AI based data mining, to provide key words, related concepts, clusters of similar ideas, etc. The traditional approach to text mining is based on searching the document and counting the number of occurrences of a given word in the document. AI-based search methods use an inductive learning algorithm to determine the key words and extract the appropriate statistical information from the textual documents $[27,42]$. An alternative text mining approach is information extraction, which finds specific information in a textual document according to a predefined set of rules and guidelines which are specific to a given topic area [69]. Commercially available text mining software packages include CRYSTAL [58], RAPIER [9], and AutopSlog [53].

On the other hand, explicit knowledge stored in the form of instances of a mathematical model (what if cases) can be leveraged via deductive and/or inductive model analysis systems. Here, deductive model analysis systems (DMAS) apply paradigmor model-specific knowledge to a single instance of the model, addressing such questions as "Why is this the solution?," "Why do the solutions to two model instances differ so much?," or, in the case of linear programming models, "Why is this instance infeasible?"' Deductive model analysis systems exist for each of the three major modeling paradigms: linear programming, simulation and spreadsheet models $[20,21,37,38,41]$.

On the other hand, inductive model analysis systems (IMAS) operate on a set of many related model instances that represent historical situations familiar to the knowledge worker and/or several (if not many) what-if cases. The primary goal of IMAS is to help the knowledge worker 
develop insight(s) into the business environment represented by the model [56]. IMAS are distinguished from deductive analysis systems by both the required input and the type of processing logic employed; i.e., IMAS apply inductive analysis technologies (e.g., statistical analysis, the group method of data handling, genetic algorithms) to extract new knowledge in the form of key factor identification, simplified meta-model generation, etc. [52,54-56, 62,65,66].

Another form of explicit knowledge leveraging is found in case-based reasoning (CBR). CBR is characterized by the knowledge worker making his or her inferences and decisions based directly on previous cases recalled from memory [40]. That is, the knowledge worker tries to avoid, or reduce, the potential for failure by recalling previous similar failures and avoiding the associated pitfalls or changing key factors in those previous failures. S/he can also speed the decisionmaking process by not having to generate and evaluate all alternatives from scratch. Finally, the attributes of past cases can be generalized to improve decision making in the future [22]. CBR requires case storage capabilities (perhaps in the form of frames), a filtering of cases for relevancy of key factors, a sophisticated recall capability based on key factors, and a case-based inference capability based on those parts of the previous case which are appropriate for the current decision.

\subsection{Learning new knowledge: converting explicit knowledge to implicit knowledge}

DSS/IT/AI can also provide valuable aids in internalizing explicit and new knowledge; i.e., in helping the knowledge worker to learn. One mode of internalizing explicit and/or new knowledge is through the modification of the internal mental model that a knowledge worker uses to serve as a performance guide in specified situations. Such mental model modifications may occur in the building of a DSS model. For example, a knowledge worker might modify his or her mental model based on the discovery of new relationships between key factors during model development, the development of counterexamples of assumed relationships, and/or the acknowledgement of fallacies in deductive logic uncovered during modeling.

Another source of mental model modification may be the adjustment of the relative importance of various components of the mental model. DMAS can be helpful here; e.g., sensitivity analysis offered in some types of mathematical modeling (i.e., linear programming models) can be used to help the knowledge worker understand and alter the relative importance of key parameters and how incremental changes in one parameter can affect the solutions [19,20].

A third source of mental model modification may come from the inductive analysis of multiple, related solved model instances. For example, if several model instances are specified in which two or more uncertain parameters are varied over appropriate ranges, an analysis of the multiple solved instances may provide new knowledge concerning not only the relative importance of key factors, but also how the key parameters interact, perhaps in a nonlinear fashion, to affect the model solution $[56,71]$.

Another aid in internalizing explicit knowledge is provided by expert systems. Here the explanation capability of ES provides understandable and amplifying rationale(s) for a recommended course of action. 
Still another way that DSS can help the knowledge worker internalize explicit knowledge is to enhance his understanding of the knowledge. Understanding, according to Perkins's [51] theory of learning, consists of knowing three things: (1) the purpose of the analysis, or what the knowledge worker wants to understand, (2) a design, or hypothesized (mathematical) model, of the process/system to be understood, and (3) arguments about why the design serves the purpose. These arguments can be of three different types. Evaluative arguments focus on the accuracy, sufficiency, necessity and consistency of a proposed model and its components. Simple explanatory arguments focus on explaining or defining the elements of the model and/or state what each element contributes. And, finally, deep explanatory arguments seek to explain a design or model in terms of basic underlying principles; e.g., the underlying formulae and interconnections between the balance sheet, income statement and funds flows statement in a business financial problem. The advantages of deep explanatory arguments include their power of abstraction, generalization, and insight generation, resulting from the application of basic principles and relations applicable to the current analysis. The basic disadvantage of deep explanatory arguments is the difficulty of defining, storing and retrieving relevant basic principles, relating these basic principles to the model, and successfully communicating the relationships to the knowledge worker [29,31,38]. Thus, this type of analysis requires not only the storage of multiple, related model cases, but also the storage, retrieval and processing of the purpose and underlying principles potentially applicable to the specific decision making environment, stored as text streams and referenced through key words and context.

\section{Goals and requirements for knowledge warehousing}

The goal of KW is to provide the decision maker with an intelligent analysis platform that enhances all phases of the knowledge management process. Several comments can be made to further amplify and explain the KW goal. First, this goal assumes that the user of the KW is the decision maker. That is, we assume that the user is not an expert in the various technologies used to enhance knowledge management, but rather is an expert in the decision making field.

Second, an intelligent analysis platform is defined as a PC-based platform that makes available to the decision maker an array of analytical tools, each of which utilizes various technologies to aid the socialization, articulation, integration, and understanding/ internalization of knowledge management. The purpose of including artificial intelligence is to amplify the cognitive capabilities of the decision maker in converting tacit knowledge into explicit knowledge, integrating this explicit knowledge by analyzing it to detect new patterns and relations, and understanding the new knowledge by providing analogs and explanations.

Third, AI technologies are often able to find important facts, patterns, relations and/or other types of new knowledge that would not have been found using standard analysis techniques such as regression analysis. The new knowledge gained can then be used to aid decision makers in determining organizational action [57]. One applicable AI technology is data mining, a process that can be divided into two distinct categories - verification-driven and discovery-driven. In verification-driven data mining, a prior hypothesis is formed about the nature of relationships among data. The result of the mining process is then used to reach a conclusion regarding the validity of this hypothesis. Discovery-driven data mining starts without any preconceived notion regarding the nature of relationships among data. It is the task of the data mining system to find 
significant patterns in the data. Two sub-categories of discovery-driven data mining are supervised learning (classification) and unsupervised learning (clustering) [15]. Supervised learning is equivalent to learning with a teacher and involves building a model for the specific purpose of optimally predicting some target field in the historical database (the value of which can be used to gauge whether the right or wrong prediction was made). In contrast, unsupervised learning does not have any well-defined goal or target to predict (and, thus, no particular supervision over what is a right or wrong answer). Techniques such as clustering and detection of association rules fall into the category of unsupervised learning [7].

The knowledge warehousing goal suggests three functional requirements for KW: (1) an ability to efficiently generate, store, retrieve and, in general, manage explicit knowledge in various forms, (2) an ability to store, execute and manage the analysis tasks and their supporting technologies with minimal interaction and cognitive requirements from the decision maker, and (3) an ability to update the KW via a feedback loop of validated analysis output. Each of these three functional requirements is discussed individually below.

\subsection{Knowledge storage and retrieval}

The KW must provide the same services for knowledge that a data warehouse provides for data. This requirement is complicated in the KW by the several different forms of knowledge feeds. That is, the primary source of data in data warehouses is transaction data (easily stored in a relational database), but the primary sources of knowledge in the knowledge warehouse include text streams from GSS and ES, film clips (stored as binary large objects or BLOBs), mathematical models and their instances (stored as equations, matrices, arc/node descriptions, etc), and analysis results (stored as equations, weight matrices, text streams, etc). Further, for knowledge stored in the form of models and solved model instances, the $\mathrm{KW}$ is required to efficiently store, retrieve and manipulate many solved model instances, with each instance tied (logically) to its associated model and/or tied (logically) to a related instance; i.e., two related instances normally exhibit a high degree of commonality in parameter values and can thus be stored and retrieved more efficiently if logically related (e.g., through inheritance).

\subsection{Analysis task management}

The analysis of knowledge is not a simple process. Specifically, an analysis task frequently utilizes various inductive and deductive AI technologies; e.g., neural networks, group method of data handling (GMDH) [6], statistics, inductive production rule generation, genetic algorithms, case-based reasoning. Each task has its own requirements with respect to (1) input data (e.g., the number and domain coverage of stored data or knowledge), (2) execution parameters required by the analysis technologies (e.g., step-size and node architecture for neural networks, the complexity factor and number of layers for GMDH), and (3) output format (e.g., weight matrix, polynomial equations, production rules, quality measures). Further, some analysis technologies are limited to specific knowledge paradigms, whereas others are equally applicable to all paradigms; e.g., the explanation task implemented in ROME/ERGO [41] is limited to spreadsheet models, whereas the meta-model generation implemented in INSIGHT is applicable to all mathematical models [56]. 
KW must efficiently support the storage, initiation, execution and management of knowledge analysis tasks and the associated implementation technologies. Specifically, the analysis tasks and the associated technologies must not only be stored in $\mathrm{KW}$, but also be logically tied to the appropriate knowledge paradigm, if required. Further, to minimize the cognitive requirements of the decision maker during analysis task execution, the required run-time interaction (e.g., appropriate step size in neural network models, complexity factors in GMDH, etc.) must be stored in the knowledge warehouse and retrieved as appropriate during task execution.

\subsection{Feedback and storage of new knowledge}

In the operation of a data warehouse, data in the warehouse is updated only periodically (say weekly or monthly) with new data from the transaction processing system. However, in the operation of a KW, the data and knowledge stored in the warehouse can be updated constantly from either of two different feedback loops: one loop associated with on-line knowledge extraction (e.g., a GSS brainstorming session), and the other loop from a real-time storage request of the decision maker/user based on the results of an analysis task s/he has validated and approved. The KW must support both feedback loops.

\section{Knowledge warehouse architecture}

These goals and requirements of a KW can be implemented via an extension of the data warehouse architecture. The proposed extension, shown in Fig. 3, consists of six major components: (1) the data/knowledge acquisition module, (2) the two feedback loops, (3) the extraction, transformation and loading module, (4) a knowledge warehouse (storage) module, (5) the analysis workbench, and (6) a communication manager/ user interface module. Each of these components is described below. 


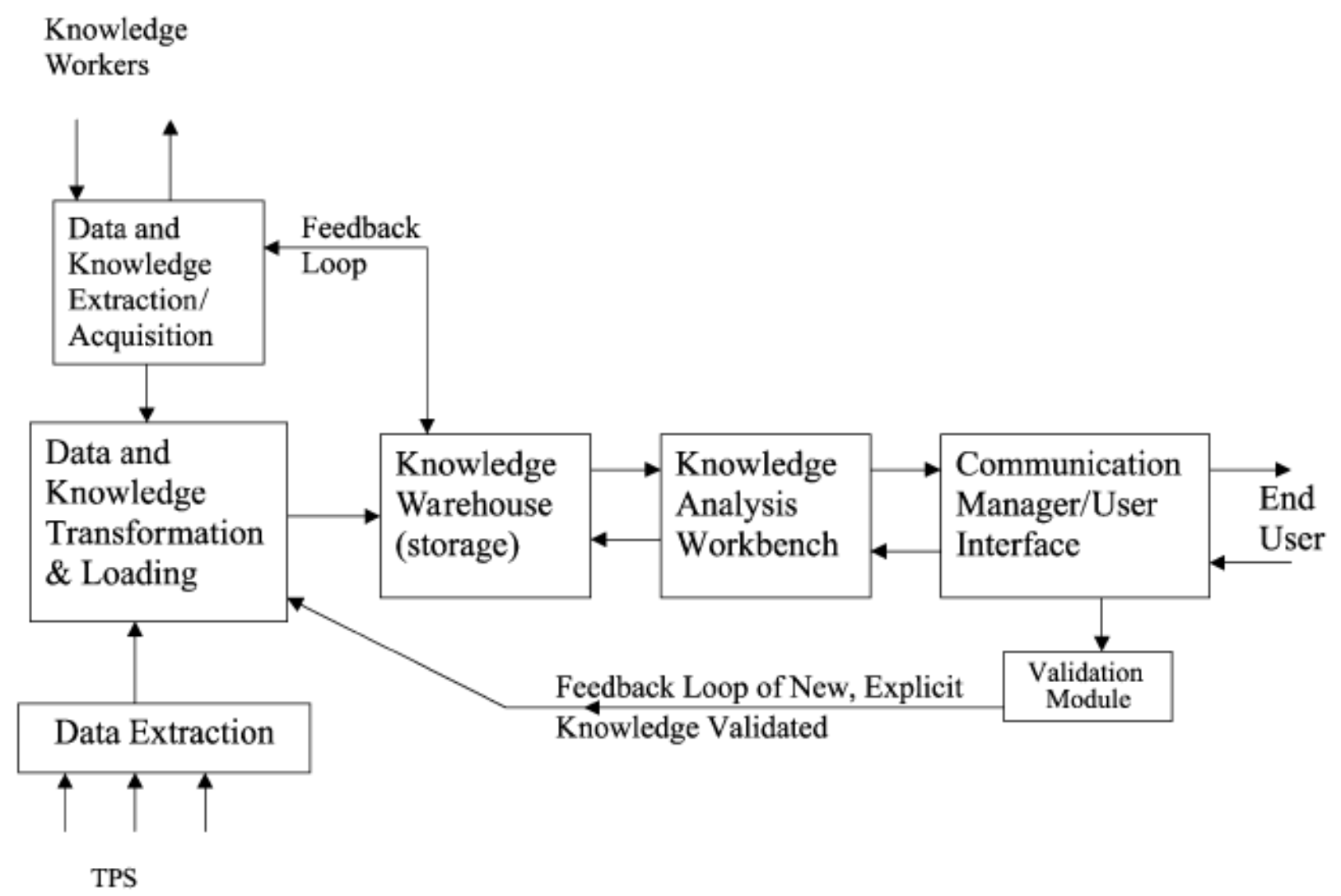

Fig. 3. Knowledge warehouse architecture.

\subsection{Knowledge acquisition module}

The knowledge acquisition module is primarily responsible for the tacit to explicit knowledge conversion; i.e., directly acquiring tacit knowledge from the decision maker/user. This acquisition module includes a specialized user interface to aid in one or more of the following processes: (1) idea generation in a GSS brainstorming environment, (2) mathematical model specification in a model-based environment, (3) what-if case specification in a model-based environment, (4) production rule elicitation in an expert system-based environment, (5) purpose and fundamental knowledge elicitation in any analysis process $[12,62]$ kinematic analysis in a physical process demonstration, etc.

\subsection{Feedback loops}

Note that there is one a feedback loop between the knowledge acquisition module and the KW storage module (via the knowledge loading module). This feedback loop provides the capability of not only storing the explicit knowledge elicited from the decision maker(s), but also of immediately broadcasting knowledge from one user to other users (in a GSS brainstorming session), displaying up-to-date lists of specified what-if cases (in a model-based DSS), or displaying current rule bases (in ES-based systems). The other feedback loop that exists between the extraction, transformation and loading module and the communication manager module 
provides for the storage of new validated explicit knowledge that has been generated in the system.

\subsection{Knowledge extraction, transformation and loading module}

The knowledge extraction, transformation and loading module is similar to that in the data warehouse in that it is responsible for extracting, reformatting, cleansing and loading data from external databases into the KW storage area (see Ref. [18]).

\subsection{Knowledge warehouse storage module}

One of the primary components of the KW architecture is an object-oriented knowledge base management system (KBMS) that integrates the knowledge base, model base, and analysis tasks. A KBMS is a system that manages the integration of a wide variety of knowledge objects into a functioning whole. These knowledge objects include numerical data, text streams, validated models, meta-models, movie clips, animation sequences, as well as the software used for manipulating them. The KBMS is implemented in an object-oriented environment. The KBMS must not only manage data, but all of the objects, object models, process models, case models, object interaction models and dynamic models used to process the knowledge and to interpret it to produce the knowledge base.

Object-specific knowledge is stored as part of the appropriate object. The specific form of the knowledge storage mechanism may include frames, semantic nets, rules, etc. Stores of knowledge include, but are not limited to, meta-data, meta-models and instances of meta-models. For example, a model's purpose is stored as part of the associated model, whereas the basic underlying principles may be stored with a more general model class.

Messages sent to the objects are generic in form, independent of the method's technology. If additional information is required to execute a specified method, a message is sent to other appropriate object(s).

The object-oriented database technology provides several advantages for this application. One advantage is that existing knowledge is integrated with (1) it own meta-knowledge, (2) examples or instances of the knowledge, and (3) methods, including the analysis tasks. This enhances storage efficiency; e.g., if the knowledge is in the form of a model and its instances, related instances may differ from a base case by only one or two parameter values and the solution vector, and all common parameter values can be inherited from the base case or other parent instance for storage efficiency. A second advantage is that some analysis tasks (e.g., the linear programming sensitivity analysis task in ANALYZE) can be logically tied to a specific class of models, whereas other analysis tasks can be tied to a super class of all models and be independent of the specific modeling paradigms. A third advantage is that method overloading allows a single user-specified command to call several different implementations of a given task and apply the appropriate technology to different forms of knowledge; this reduces the cognitive burden on the decision maker by providing him/her with independent execution calls (i.e., messages) for all analysis tasks. It also provides a primary prerequisite for effective management 
of technology; i.e., overloading, in conjunction with encapsulation, makes the changing of implementation technologies transparent to the user.

\subsection{Knowledge analysis workbench}

The analysis workbench handles all interaction with the analysis tasks, including task control, argument generation, and management of technology. The task controller handles all requests for data and run-time interactions (e.g., complexity factors in GMDH algorithms, step sizes in neural networks) required by the analysis technologies. That is, the task controller acts as an AI-based surrogate decision maker for task interactions, shielding the real decision maker from the requirements of knowing the technologies, their nuances, interactions, etc.

The argument generation sub-module evaluates the outputs of the various analysis tasks, especially the causation task, filtering out implausible or inconsistent results based on relative measures of accuracy, simplicity, conceptual validity, sufficiency, necessity, and consistency. It then generates simple and deep explanatory arguments that (hopefully) enhance the decision maker's understanding of the modeled environment. In generating these arguments, the argument generation module interfaces with the knowledge base, the instance base and model base, applying deductive knowledge, analogical reasoning, and other technologies, as appropriate.

The management of technology module manages the repository of analysis technologies. Specifically, it provides for the encapsulation of new analysis algorithms into object model classes, integration of legacy data mining applications, incorporation of new analytical models and meta-models into the object model repository, etc.

\subsection{Communication manager}

This module, which handles all analysis communication between KBMS and the user interface, includes six functional sub-modules: a knowledge engineer, what-if interface, query processor, results presentation manager, on-line help, and user interface.

The knowledge engineer sub-module is an expert system-based sub-system responsible for interacting with the decision maker to develop the purpose of the analysis and the basic underlying principles of the modeled environment. Both types of knowledge are used in the development of arguments. This knowledge may be stored in the knowledge base in the form of frames, rules, semantic nets, etc.

The what-if interface is designed to efficiently and effectively help the decision maker specify one or more what-if cases to be investigated. It includes an analogical component that is used to suggest pertinent instances by varying one or more parameter values. It also includes one or more interactive graphical displays, or summaries, of instances already available, so that the decision maker can see at a glance what has already been tried and what instance(s) might lead to additional insights. The what-if interface also includes a capability to suggest potentially valuable cases based on the planning analysis task. 
The query processor provides the interface between the decision maker and the analysis task. It translates natural language, QBE or SQL-like queries specified by the decision maker into machine executable queries.

The result representation manager selects the most appropriate presentation view for each analysis result; e.g., graphics [50], natural language production rules, polynomials, decision trees, etc. The selection is based on a combination of the analysis task output and the decision maker's preference which, in turn, is based on an adaptable machine learning algorithm which analyzes previous uses of models and analysis tasks by the current decision maker [14,44].

The help sub-module provides the user with information concerning the model (e.g., assumptions, parameter ranges, units of measurement, internal model structure), instances (differences from base case, key decision variable values), pertinent knowledge (e.g., metamodels, meta-data, basic principles, analysis purpose), and analysis tasks (e.g., applicable technology, technology description, explanatory traces of results, technical parameters used, advantages and limitations of technologies).

\section{Development and implementation of the knowledge warehouse architecture}

Development and implementation KW architecture outlined earlier may involve considerable amount of organizational time and effort and may cross the boundaries of many business units and departments. The usual time frame is measured in months and not days and the amount of money involved usually represents millions not thousands of dollars. As with the development and implementation of DSS projects [1], [2], [3], [4], [45],[59], [60] and [68], a large-scale KW project may require large investment of time and money that puts a tremendous pressure on those involved. As a result, KW projects may not always be successful. Many of the factors that affect the successful development and implementation of DSS projects can also be important in determining success for $\mathrm{KW}$. Among these factors are support from the top executives [24], [43] and [45]; users involvement and participation [5], [30], [31], [32], [61] and [67]; well-defined business objectives or goals for the DSS [45], [61] and [68]; resources adequacy issues; organization and political issues within the company [17], [43] and [70]; technological issues [45], [61] and [68]; process management issues [47] and [49]; goals, plans and communication issues [25]; values and ethics [33] and other external issues.

All of these factors play an important role in successful development and implementation of knowledge warehouses. However, since knowledge warehouses focus on the harnessing of intellectual capital within an organization and making it available to all who need them, additional factors should be considered as well. These factors are due to the additional tasks that knowledge warehouses should perform. They are the following.

(1) Creation of a knowledge management infrastructure. The task involves workstations, networks, databases, search engines, and publishing tools.

(2) Building a knowledge culture by active promotion of the knowledge agenda, including the development and diffusion of knowledge management models, frameworks, and language. This 
requires the creation of mechanisms for the development and maintenance of knowledge bases in different functions and departments.

(3) Facilitation of knowledge-oriented connections, coordination and communication throughout, and also without, the organization.

Successful development and implementation of $\mathrm{KW}$ architecture requires these generic activities.

1. Designing and implementing techniques to identify and record both knowledge and ignorance (e.g., taking inventory and auditing) and then designing processes to share, use and protect such knowledge and to remedy ignorance by learning or knowledge creation.

2. Designing and orchestrating contexts, environments and activities to discover and release what is not formally or explicitly known (e.g., socializing and experiencing) and possibly coaching and encouraging people to be effective in these processes.

3. Articulating and communicating the purpose and the nature of knowledge management and connecting it to other strategic and operational initiative and activities of the organization. Although it is beyond the scope of the paper to develop a prototype for the proposed KW architecture, we provide a list of vendors and their products that can be employed to implement the modules of the KW architecture. There are plethora of products available commercially and the list we provide here is not exhaustive by nature. In addition, our goal was not to identify the best vendor or the best product that is available to aid the $\mathrm{KW}$ architecture, but rather to review and present some product offerings that support the various processes of the proposed architecture. To identify the vendors/products, we reviewed several sources such as, KM World, Knowledge Management, KM World Buyer's Guide, Directory of Data Warehousing solution providers, and by searching the web on Knowledge Management and Data Warehousing related product offerings. In reviewing the product offerings, we found that although many products cover each component of the KW architecture individually, there were very few that provided an integrated solution covering all aspects of the KW. (See Exhibit A for a partial list of products that claim to offer integrated solution for the KW architecture.)

There are many other products offered by vendors that may not provide a comprehensive solution but support the implementation of the processes under each component of the KW architecture that we have proposed.

The KW should efficiently generate, store, retrieve and, in general, manage explicit knowledge in various forms to provide the decision maker with an intelligent analysis platform that enhances all phases of knowledge,. The knowledge based systems module in our proposed KW architecture helps accomplish that and tools for this module should support processes such as mental model extraction, knowledge engineering and integration for the extraction and storage of various types of organizational knowledge. (Some examples of vendors and their products available to support the KBMS module of KW architecture can be found in Exhibit A.) Secondly, the KW should be able to store, execute and manage the analysis tasks and its supporting technologies. Processes such as sensitivity analysis, mining of the knowledge warehouse, machine learning and pattern recognition fall under this component. In addition, capabilities such as hypothesis testing for meta-models should also be available. (See Exhibit 
A for examples of vendors and their products available to support the Knowledge Analysis Workbench module of KW architecture.)

Finally, the KW should provide computer-assisted support to generate natural language arguments concerning both the comparable validity of the models, meta-models and relations produced by analysis tasks, and how this new knowledge relates to the decision maker's purpose. (See Exhibit A for a sample of vendors and their products that can support communication manager module of our KW architecture.)

\section{Roadmap for future DSS research}

In general, DSS has made significant research contributions in knowledge extraction/acquisition with knowledge engineers of expert systems and the mathematical models of management scientists. DSS has also made significant contributions in the warehousing of data/knowledge and in the communication of results to end users; i.e., databases and user interfaces are principle components of all DSSs.

However, the knowledge spiral proposed by Nonaka and Takeuchi [48] along with the knowledge warehouse proposed herein suggest a different direction for DSS in the next decade. This new direction is based on an expanded purpose of DSS; specifically, the purpose of DSS should be to enhance all four aspects of the knowledge spiral (tacit to tacit knowledge sharing, tacit to explicit knowledge conversion, new knowledge generation, and explicit to tacit knowledge internalization). That is, the purpose of DSS is knowledge enhancement.

In this vein, one research area of DSS becomes the development of a set of theoretical foundations upon which to build future development and applications, one for each quadrant of the knowledge spiral. For instance, cognitive mapping might provide a productive foundation for the tacit to explicit knowledge conversion as a source of study for extracting and analyzing the decision maker's mental models of a given decision making environment. Similarly, the Gestalt theory of insight [39] combined with Newell and Simon's [46] theory of goal directed search might provide a solid foundation for new knowledge generation, based on the dichotomy of inductive/deductive problem solving and reflecting the hemispheric specificity of the human brain. Cognitive dissonance and Perkin's [51] theory of understanding might provide a valid foundation for explicit to tacit knowledge internalization, the former suggesting that no learning takes place until the decision maker perceives a significant difference between his/her mental model and the real world, and the latter suggesting ways to resolve such perceived differences. And finally, the theory of communication might provide a general foundation for tacit to tacit knowledge sharing.

This expanded purpose of DSS as knowledge enhancement also suggests that the effectiveness of each DSS will, in the future, be measured based on how well it promotes and enhances knowledge, how well it improves the mental model(s) and understanding of the decision maker(s) and thereby how well it improves his/her decision making. One research thrust along these lines, especially applicable to model-based DSS, might include extracting (via case analysis) the novice decision maker's mental model in some decision making environment, comparing it to the (expert's) mathematical model, generating arguments concerning why the 
mathematical model is superior to the decision maker's mental model, feeding these arguments back to the decision maker in an attempt to change and improve his/her mental model, and then re-testing the decision maker to determine whether his/her revised mental model produces better decisions. Such a research thrust, based on the lens model [8] and [36], would provide a missing link in the current research [13].

This expanded purpose of DSS also points out that one of the four quadrants of the knowledge spiral, specifically the tacit to tacit knowledge sharing, has been largely ignored in the DSS literature. This suggests potentially viable research directives in both kinematics for learning physical actions concerned with decision making and a kinematics equivalent for learning-bydoing or on-the-job-training in mental model formulation. Research questions that might be addressed include: How decision making is learned (i.e., by observation, by case analysis, etc.), How many observations or cases are required to learn complex tasks, How transferable such knowledge is, etc.

In addition to the expanded purpose of DSS, the knowledge warehouse architecture proposed in this article shows two major areas in which DSS could/should foster future research and development. One such area is in providing the motivation, tools, techniques and demonstrated benefits associated with the development and use of the knowledge analysis workbench. In the DSS literature, especially the management science aspects of it, the focus of research has historically been on model specification and model solution. In the future, it seems that the analysis of solutions is the more important aspect of modeling, along with providing the decision maker with an understanding of the analysis results. Several DSS researchers have developed some theory in this vein, but the area still needs further refinement. For example, in model-based DSS, we need to identify a 'minimal spanning set' of analysis tasks that leads to successful model analysis, and to validate these tasks through experimentation.

Another research area could explore and evaluate technologies that are potentially applicable to analysis and understanding. Initial evaluation could match the input, processing, output, and feedback characteristics of various technologies against the corresponding requirements of the prime analysis tasks mentioned above. The results would provide a research agenda for the application of the technologies to the analysis tasks, along with empirical testing of their effectiveness.

A third research area would utilize artificial intelligence techniques to develop deep explanatory arguments based on basic principles and organizational goals to show why one suggested decision is 'better' than comparable alternatives in a given decision making environment. Such deep explanations could improve the decision maker's confidence in the DSS, as well as enhance his/her insight into the decision making environment and foster better decisions in the future. It should be noted, however, that this requirement assumes the existence of a knowledge warehouse containing the basic business principles and the organizational goals, as well as an indexing scheme and search algorithms to extract appropriate principles and goals for specific arguments.

A second area in the knowledge warehouse architecture that could benefit from future DSS research is in the validation process of knowledge prior to being fed back into the knowledge 
warehouse. Such questions that should be addressed include: (1) How much filtering of potential new knowledge should be allowed, (2) Who should be responsible for this filtering (CKO, leaders in GSS/GDSS, etc.), (3) What the filtering criteria should be, and (4) What are the tradeoff of artificial intelligence vs. human intelligence in this filtering process. The answers to these questions could significantly impact the implementation and eventual overall quality of the knowledge warehouse and the decisions it supports.

\section{Summary and conclusions}

In this paper, we have proposed a knowledge warehouse $(\mathrm{KW})$ architecture as an extension to the Data Warehouse (DW) model. The KW architecture will not only facilitate the capturing and coding of knowledge but will also enhance the retrieval and sharing of knowledge across the organization. Essentially, the KW will provide the same service for knowledge that a DW provides for data. The primary goal of the KW is to provide the decision maker with an intelligent analysis platform that enhances all phases of knowledge.

The development and implementation of the KW architecture proposed here is a large, multifaceted project, with much work remaining. Specifically, there are three major aspects of associated research. The first addresses the analysis tasks themselves; specifically, (1) defining/refining the analysis tasks that most likely enhance insightful understanding, (2) developing a task-vs.-technology table that matches the various inductive analysis technologies with the appropriate analysis task, and (3) evaluating the results of these technologies when applied to model analysis. The second area of research addresses the empirical testing of the insight generation capability of $\mathrm{KW}$ and its analysis tasks in both a controlled and real-world environment. A third area of research addresses the computer-assisted generation of arguments, especially deep explanatory arguments, and empirically testing their ability to enhance user understanding.

In order to accomplish these goals, the KW should efficiently generate, store, retrieve and, in general, manage explicit knowledge in various forms. Secondly, the KW should be able to store, execute and manage the analysis tasks and it's supporting technologies. Finally, the KW should provide computer-assisted support to generate natural language arguments concerning both the comparable validity of the models, meta-models and relations produced by analysis tasks, and how this new knowledge relates to the decision maker's purpose.

The proposed KW architecture consists of an object-oriented knowledge base management system module (OO-KBMS), a knowledge analysis workbench, and a communication manager. The OO-KBMS module integrates a wide variety of knowledge objects and analysis tasks. The knowledge analysis workbench handles the interaction with the analysis tasks, including task control, argument generation, and encapsulation of new analysis algorithms into object models. The communication manager handles all analysis communication between the OO-KBMS and the user interface. The communication manager accomplishes this effectively through the use of five functional sub-modules: a knowledge engineer, what-if interface, query processor, results presentation manager, and on-line help. 
The KW will also include a feedback loop to enhance its own knowledge base with the passage of time, as the tested and approved results of knowledge analysis is fed back into the $\mathrm{KW}$ as an additional source of knowledge. The primary role of the feedback loop is to provide the capability of both storing the explicit knowledge elicited from the decision maker(s), and also immediately making it available for other users in the system.

\section{Exhibit A.}

Products that claim to offer integrated solution for the KW architecture are Raven, FrontOffice and Knowledge Warehouse 5.0 from SAP.

- Raven is the code name for the package released in mid 2000 by Lotus Development which has three main components - expertise profiling/locating, a collaborative portal and content tracking and analysis piece (Velker 1999).

- FrontOffice Technologies' flagship product FrontOffice's capabilities include: an enterprise document management based on Microsoft Exchange; integrated searching of enterprise documents, e-mail, intranet, and the internet; and document access from the FrontOffice Workplace, Microsoft Exchange, Windows 95 Explorer, and custom applications.

- SAP's Knowledge Warehouse 5.0 offers five different functionalities-Web check-in, authoring and editing, Document Modeling Workbench, Performance Assessment Workbench, Integration with the Document Management System (DMS), Connection to business workflows.

The following are some examples of vendors and their products available to support the KBMS module of the proposed KW architecture.

(a) Tower Technology (www.towertechnology.com) delivers high volume, production imaging, case management and integrated document management (IDM) solutions. The flagship product Tower IDM is an integrated document management solution that is tightly integrated with Lotus Notes and MS exchange. Tower IDM provides one common enterprise for full function production imaging, case management, COLD/ERM and document management in both client/server and internet browser environments.

(b) IBM software Solution's (www.software.ibm.com/data) KnowledgeX enables companies to make informed decisions by improving the creation, dissemination and use of acquired organizational knowledge. IBM's KnowledgeX facilitates conversion of information into knowledge by revealing hidden relationships from disparate information sources.

(c) FileNET (www.filenet.com) delivers content management software solution for corporate and government organizations. FileNET's Panagon products help customers to better manage their digital content and business processes in order to use information more effectively. FileNET's internet and client/server solution provide standard-based workflows, document imaging, electronic document managing and report management (Computer Output to Laser Disk (COLD)) software for managing information and enhancing productivity. 
(d) The Unisys (www.unisys.com) Universal Repository (UREP), a highly scaleable enterprise system, helps integrate different services (such as Asset Management, Component-based Development, Corporate Meta Data Management, and Tool Interoperability) of the enterprise. The following are some examples of vendors and their products available to support the knowledge analysis workbench module.

(a) The VantagePoint (www.thevantagepoint.com): provides competitive technical intelligence professionals and technology managers with new, powerful, and unique capabilities to help extract knowledge from text databases thus enhancing the following five analysis tasks:

-scanning (identification of new technologies, developments in existing technologies, and new uses of technologies),

•profiling (discovery of the key people and organizations),

-mapping and decomposition (identification of key dependency relationships among technologies (other technologies, scientific phenomena, manufacturing capabilities, etc.)),

-trending (establishing how a technology has emerged, its applications, and what factors (technical and non-technical) appear to govern its development),

-forecasting (projecting how a technology could evolve, how it might diffuse into application, and the potential impacts of these events).

(b) VxInsight (http://www.cs.sandia.gov/ dkjohns/JIIS/Vx_Overview.html): developed by Sandia National Laboratories, VxInsight provides a visual mechanism for browsing, exploring and retrieving information from a database. The graphical display conveys information about the relationship between objects in several ways and on multiple scales. In this way, individual objects are always observed within a larger context.

(c) KnowledgeMiner (http://www.knowledgeminer.net/): is a new data-mining tool that enables anyone to use its unique form of modeling to quickly visualize new possibilities. It uses principles of Artificial Intelligence and the tool is designed to extract hidden knowledge from data easily. It was built on the cybernetic principles of self-organization: Learning a completely unknown relationship between output and input of any given system in an evolutionary way from a very simple organization to an optimally complex one.

(d) Dataware Technologies' Knowledge Management Suite 3.0 (www.dataware.com) with its text mining capabilities helps users discover hidden relationships between concepts that are buried in large knowledge sources. It accomplishes this by generating a list of related concepts thus increasing the amount of information users can process and at the same time minimizing the possibility of overlooking key information. It provides a single point access to internal and external data sources. It also helps identify and contact co-workers with expertise on specific topics. 
(e) Autonomy (www.autonomy.com) develops software that automates large volumes of unstructured content. It is able to automate these tasks because of the software's ability to analyze a document, extract ideas, and determine which ideas are most important. This is the result of proprietary pattern matching technology. The software can also profile users by analyzing the ideas in the document they read or produce. Autonomy's Portal-in-a-box features the ability to automatically create and maintain easy to navigate portal with well-organized information from hundreds of sources.

The following are some examples of vendors and their products available to support the communication manager module.

(a) 80-20software (www.80-20.com): The document management extensions for Microsoft exchange delivers ubiquitous, seamless and inexpensive document management to the enterprise. MS exchange 5.0/5.5, office 97, outlook 97, windows 95/NT4.0 and internet explorer should be available to every user in the organization with the power to share information.

(b) Lotus Development's (www.lotus.com) Domino.Doc has transformed document management from a niche application for small groups of specialists to a broader, flexible infrastructure solution, scalable to every user across the organization. The fact that it is fully customizable enables an organization to manage documents throughout their life cycle, share info across the network via web browsers, notes other applications. It also leverages the scalability, flexibility and security of the Lotus Domino server and thus functions as a key component of knowledge management through the enterprise.

(c) Knowledge Track's (www.knowledgetrack.com) corporate portal solution is the Knowledge Center v3.0. Often corporations use departments as pilots for implementation of corporate portals. Although a solution may be successful in the departmental level, the challenge is to take that solution and spread it in the enterprise. Typical problems are lack of scalability and sluggish performance. The knowledge Center offers a central location for employees to unlock and organize corporate information relevant to their job functions and thus help companies to compete more effectively. The enterprise can share information with the entire supply-chain, collaborate around information, and easily view and search for information.

\section{References}

[1] T Ahn, G Grudnitski. Conceptual perspectives on key factors in DSS development: a systems approach. Journal of Management Information Systems, 2 (1) (1985), pp. 18-32 Summer

[2] M Alavi, E Joachimsthaler. Revisiting DSS implementation research: a meta-analysis of the literature and suggestions for researchers. MIS Quarterly, 16 (1) (March 1992), pp. 95-116

[3] S Alter. Transforming DSS jargon into principles for DSS success. P Gray (Ed.), Decision Support and Executive Information System, Prentice-Hall, Englewood Cliffs, NJ (1994), pp. 226 
[4] S Alter. What do you need to know to develop your own DSS? P Gray (Ed.), Decision Support and Executive Information System, Prentice-Hall, Englewood Cliffs, NJ (1994), pp. 5865

[5] H Barki, J Hartwick. Measuring user participation, user involvement, and user attitude MIS Quarterly, 18 (1) (March 1994), pp. 59-82

[6] A.R Barron. Predicted square error: a criterion for automatic model selection S.J Farlow (Ed.), Self-Organizing Methods in Modeling: GMDH Type Algorithms, Marcel Dekker, New York (1984), pp. 86-104

[7] A Berson, S Smith. Data Warehouse, Data Mining, and OLAP, McGraw-Hill, New York (1997)

[8] E Brunswik. The Conceptual Foundation of Psychology, University of Chicago Press, Chicago, IL (1952)

[9] M.E. Califf, Relational Learning Techniques for Natural Language Information Extraction, PhD Proposal, Department of Computer Sciences, University of Texas at Austin, 1997.

[10] C.W Choo. The Knowing Organization: How Organizations Use Information to Construct Meaning, Create Knowledge, and Make Decisions, Oxford, New York (1998)

[11] T Davenport, L Prusak. Working Knowledge, Harvard Business School, Boston (1998)

[12] B Devlin. Data Warehouse: From Architecture to Implementation, Addison Wesley Longman, Menlo Park, CA (1997)

[13] J.S Dhaliwal, I Benbasat. The use and effects of knowledge-based system explanation: theoretical foundations and a framework for empirical evaluation. Information Systems Research, 7 (3) (September 1996), pp. 342-362

[14] D.R Dolk, B Konsynski. Knowledge representation for model management systems. IEEE Transactions of Software Engineering, 10 (6) (1984), pp. 619-628

[15] U.M Fayyad, G Piatetsky-Shapiro, P Smyth, R Uthurusamy. Advances in Knowledge Discovery and Data Mining, AAAI Press, Menlo Park, CA (1996)

[16] R Fourer. Modeling languages versus matrix generators. ACM Transactions on Mathematical Software, 9 (2) (1983), pp. 143-183

[17] J.E Gaskin. Corporate Politics and the Internet: Connection Without Controversy, PrenticeHall, Upper Saddle River, NJ (1997)

[18] P Gray, H Watson. Decision Support in the Data Warehouse, Prentice-Hall, New Jersey (1998) 
[19] H.J Greenberg. A Computer-Assisted Analysis System for Mathematical Programming. Models and Solutions: A User's Guide for ANALYZE, Kluwer Academic Publishing, Boston, MA (1993)

[20] H.J Greenber. Enhancements of ANALYZE: a computer-assisted analysis system for linear programming. ACM Transactions on Mathematical Software, 19 (2) (1993), pp. 223-256

[21] H.J Greenberg. Syntax-directed report writing in linear programming using ANALYZE European Journal of Operational Research, 72 (1994), pp. 300-311

[22] K.F Hammond. Case-based planning. Proceedings of Workshop on Case-Based Reasoning (May 10-13, 1988), pp. 17-20

[23] K. Harris, Chief Knowledge Officer: Managing Intellectual Assets, Gartner Group Document, \#KA-03-8437, Boston, MA, March 1998.

[24] J.T Hogue, H.J Watson. Management's role in the approval and administration of DSS. MIS Quarterly, 7 (2) (June 1983), pp. 15-26

[25] B.R Horner, I Benbasat. Measuring the linkage between business and information technology objectives. MIS Quarterly, 20 (1) (March 1996), pp. 55-81

[26] W.H Inman. Building the Data Warehouse, Wiley, New York (1996)

[27] D.-H Jang, S.H Myaeng. Development of a document summarization system for effective information services. RIAO 97 Conference Proceedings: Computer-Assisted Information Searching on Internet, Montreal, Canada (1997), pp. 101-111

[28] L Johnson. The need for competence models in the design of expert systems. International Journal in Systems Research and Informational Science, 1 (1985), pp. 23-36

[29] C.V Jones. An introduction to graph based modeling systems: Part I. Overview. ORSA Journal On Computing, 2 (2) (1990), pp. 136-151

[30] K Joshi. The measurement of fairness or equity perceptions of management information systems users. MIS Quarterly, 13 (3) (September 1989), pp. 343-358

[31] K Joshi. A model of users' perspective on change: the case of information systems technology implementation. MIS Quarterly, 15 (2) (June 1991), pp. 228-241

[32] K Joshi, T Lauer. Impact of information technology on users' work environment: a case of computer aided design (CAD) system implementation. Information and Management, 34 (1998), pp. $349-360$ 
[33] E.A Kallman, J.P Grillo. Ethical Decision Making and Information Technology (2nd edn.), McGraw-Hill, New York (1996)

[34] J.L Kennington, R.V Helgason. Algorithms for Network Programming, Wiley, New York (1980)

[35] A.L Kidd. Knowledge Acquisition for Expert Systems: A Practical Handbook, Plenum, New York (1987)

[36] C.N Kim, R McLeod Jr. Expert, linear models and nonlinear models of expert decision making in bankruptcy prediction: a lens model analysis. Journal of Management Information Systems, 16 (1) (Summer 1999), pp. 189-206

[37] S.O Kimbrough, C.W Pritchett, M.P Bieber, H.K Bhargava. The coast guard's KSS project. Interfaces, 20 (6) (1990), pp. 5-16

[38] S.O Kimbrough, J.R Oliver, C.W Prichett. On post-evaluation analysis: candle-lighting and surrogate models. Interfaces, 23 (3) (1993), pp. 17-28

[39] W Kohler. The Task of Gestalt Psychology, Princeton Univ. Press, Princeton, NJ (1969)

[40] J.L Kolodner. Extended problem solver capabilities through case-based inference.

Proceedings of a Workshop on Case-Based Reasoning (May 10-13, 1987), pp. 21-30

[41] D.W Kosy, B.P Wise. Self-explanatory financial planning models. Proceedings of the National Conference of Artificial Intelligence (August 1984), pp. 176-181

[42] J Kupiec, J Pedersen, F Chen. A trainable document summarizer. E.A Fox, P Ingwersen, R Fidel (Eds.), SIGIR-95: Proceedings of the 8th Annual International ACM SIGIR Conference on Research and Development in Information Retrieval, ACM press, New York, NY (1995), pp. 68-73

[43] A.L Lederer, V Sethi. Root causes of strategic information systems planning implementation problems. Journal of Management Information Systems, 9 (1) (Summer, 1992), pp. $25-46$

[44] T.P Liang. Model management for group decision support. MIS Quarterly (December 1988), pp. 667-680

[45] J.R Meredith. The implementation of computer bases systems. Journal of Operational Management (October 1981), pp. 11-21

[46] A Newell, H.A Simon. Human Problem Solving, Prentice-Hall, Englewood Cliffs, NJ (1972) 
[47] M Newman, R Sabherwal. Determinants of commitment to information systems development: a longitudinal investigation. MIS Quarterly, 20 (March 1996), p. 1

[48] I Nonaka, H Takeuchi. The Knowledge-Creating Company, Oxford Univ. Press, New York (1995)

[49] S Palvia, N.L Chervany. An experimental investigation of factors influencing predicted success in DSS implementation. Information and Management, 29 (1995), pp. 43-53

[50] D Parsaye, M Chigness, S Khoshafian, H Wong. Intelligent databases. AI Expert (March 1990), pp. 38-47

[51] D.N Perkins. Knowledge as Design, Lawrence Erlbaum Associate, Hillsdale, NJ (1986)

P.C Piela, T Epperly, K Westerberg, A Westerberg. An object-oriented computer environment for modeling and analysis, Part 1 -the modeling language. Computers and Chemical Engineering, 15 (1) (1991), pp. 53-72

[53] E Riloff. Automatically generating extraction patterns from untagged text. Proceedings of the Thirteenth National Conference on AI (1996), pp. 1044-1049

[54] A Saltelli, T Homma. Sensitivity analysis for model output. Computational Statistics and Data Analysis, 13 (1992), pp. 73-94

[55] A Saltelli, J Marivoet. Non-parametric statistics in sensitivity analysis for model output: a comparison of selected techniques. Reliability Engineering and Systems Safety, 28 (1990), pp. $229-253$

[56] R Sharda, D.M Steiger. Inductive model analysis systems: enhancing model analysis in decision support systems. Information Systems Research, 7 (3) (1996), pp. 328-341

[57] D.J Skyrme, D.M Amidon. Creating the Knowledge-Based Business, Business Intelligence, London (1997)

[58] S Soderland, W Lehnert. Wrap-up: a trainable discourse module for information extraction. Journal of Artificial Intelligence Research, 2 (1995), pp. 131-158

[59] R Sprague. DSS in context. R Sprague, H Watson (Eds.), Decision Support Systems:. Putting Theory into Practice (2nd edn.), Prentice-Hall, Englewood Cliffs, NJ (1989)

[60] R Sprague, E Carlson. Building Effective Decision Support Systems, Prentice-Hall, Englewood Cliffs, NJ (1982)

[61] R.H Sprague, H.J Watson. Decision Support for Management, Prentice-Hall, Upper Saddle River, NJ (1996) 
[62] D.M Steiger. Enhancing user understanding in a decision support system: a theoretical basis and framework. Journal of Management Information Systems, 15 (2) (Fall, 1998), pp. 199-221

[63] D.M Steiger, R Sharda. LP modeling languages for personal computers: a comparison. Annals of Operations Research, 43 (1993), pp. 195-216

[64] D.M Steiger, R Sharda, B LeClaire. Graphical interfaces for network modeling: a model management system perspective. ORSA Journal On Computing, 5 (3) (1993), pp. 275-291

[65] G Stephanapoulos, G Henning, H Leone. MODEL.LA — a modeling language for process engineering-I. The formal framework. Computers and Chemical Engineering, 14 (8) (1990), pp. 813-846

[66] G Stephanapoulos, G Henning, H Leone. MODEL.LA — a modeling language for process engineering: II. Multifaceted modeling of processing systems. Computers and Chemical Engineering, 14 (8) (1990), pp. 847-869

[67] P Tait, I Vessey. The effect of user involvement on system success. MIS Quarterly, 12 (1) (1988), pp. 91-108

[68] E Turban. J Aronson, Decision Support Systems and Intelligent Systems (6th edn.). Prentice-Hall, Upper Saddle River, NJ (2000)

[69] P. Turney, Extraction of keyphrases from text: evaluation of four algorithms, Technical Report, National Research Council of Canada, 1997.

[70] B Tuttle. Investigating the impacts of managerial turnover/succession on software project performance. Journal of Management Information Systems, 9 (2) (Fall, 1992), pp. 127-144

[71] H.M Wagner. Global sensitivity analysis. Operations Research, 43 (6) (1995), pp. 948-969

[72] L Wah. Behind the Buzz. Management Review (April 1999), pp. 16-26 